\title{
An artificial immune system with continuous-learning for voltage disturbance diagnosis in electrical distribution systems
}

\author{
Fernando P.A. Lima, Mara L.M. Lopes, Anna Diva P. Lotufo, Carlos R. Minussi* \\ Electrical Engineering Department, Faculty of Engineering of Ilha Solteira (FEIS), UNESP, Univ Estadual Paulista "Júlio de Mesquita Filho", Av. Brasil 56, PO \\ Box 31, 15385-000 Ilha Solteira, SP, Brazi
}

\section{A R T I C L E I N F O}

\section{Article history:}

Received 6 November 2014

Revised 21 January 2016

Accepted 6 March 2016

Available online 15 March 2016

\section{Keywords:}

Diagnosis

Voltage disturbances

Artificial immune systems

Negative selection algorithm

Clonal selection algorithm

Continuous-learning

Electrical distribution systems

\begin{abstract}
A B S T R A C T
This paper presents a new artificial immune algorithm with continuous-learning, which is inspired by the biological immune system, to realize the voltage diagnosis in electrical distribution systems. This conception allows one to compose a diagnosis system that can continuously learn without reinitialization when new disturbances occur due to the evolution of the electrical system. Two artificial immune algorithms, which are the negative selection algorithm and the clonal selection algorithm, are used for the pattern recognition process and the learning process, respectively. The principal application of this new method aids the operation during failures, supervises the protection system, and can evolve with the power systems to continuously acquire new knowledge. This new methodology has a direct impact in the area of diagnosis in electrical systems, as well as, in the pattern recognition problem, because the main contribution and novelty of this method is the continuous learning capability, which enables the system to learn unknown patterns without having to restart the knowledge. This is the major advantage of this methodology. To evaluate the efficiency and performance of this new method, failure simulations were performed in a real distribution system with 134 buses using the EMTP software. The results show robustness and efficiency.
\end{abstract}

(c) 2016 Elsevier Ltd. All rights reserved.

\section{Introduction}

Nowadays, a new concept of electrical power systems called Smart Grids requires several investments to transform the electrical systems that provide modern technologies to generate, transmit and principally distribute electrical energy systems (Dongli, Meng, \& Song, 2011), (Gungor et al., 2011). The smart grid concept is based on an intense usage of automation, computation and telecommunication technologies to monitor and control electrical systems that allow the implementation of new control, communication, protection and optimization strategies to improve the efficiency of the currently available systems (Gungor et al., 2011).

Thus, several technologies are used, particularly digital and information technology (Alag et al., 2001), which allow the development of integrated systems that combine acquisition, analysis and data processing techniques, to provide the necessary assistance to automation, control and decision-making processes (NorthcoteGreen \& Wilson, 2007).

\footnotetext{
* Corresponding author. Tel.: +5501837431225.

E-mail addresses: engfernandoparra@gmail.com (F.P.A. Lima), mara@mat.feis.unesp.br (M.L.M. Lopes), annadiva@ieee.org (A.D.P. Lotufo), minussi@dee.feis.unesp.br, crminussi@gmail.com (C.R. Minussi).
}

Considering the failure diagnosis for the smart grid, those systems must be based on auto-restoration idea, i.e., they must be able to automatically detect, analyze, respond and restore failures (Dongli et al., 2011) without human intervention. Thus, using intelligent techniques (artificial neural networks, fuzzy logic, artificial immune systems, etc.) is an alternative to the diagnosis problem. Several techniques based on this concept have been used to aid the operators in executing routines in electrical systems, which provides security, velocity and efficiency in planning corrective actions.

However, most failure-diagnosis methods based on intelligence proposed to the actual electrical systems have a learning strategy to obtain the knowledge, on which all efficiency and robustness depend. Normally, these strategies (training or learning algorithms) must always reinitialize the learning process when the system is executed or a new disturbance occurs; therefore, the system is not significantly intelligent. The system does not constantly learn, and when a new pattern appears, every learning process is executed again.

A modern and intelligent failure-diagnosis system to the smart grids must continuously learn, follow innovations and modernizations of the electrical system, which include new types of prominent failures, learn with experience and constantly evolve. 
Developing a system with these characteristics is a complex task. In the literature, many studies present methods to solve the problem of the diagnosis of disturbances, as in (Uyar, Yildririm, \& Gencoglu, 2008), (Oleskovicz et al., 2009), (Zhang, Li, \& Hu, 2011), (Lima, Lotufo, \& Minussi, 2014), (Lima, Minussi, Bessa, \& Fidalgo, 2015), however these approaches do not consider the minimum requisites to the smart grids, i.e., the objective to provide continuous-learning to a pattern recognition system. This requisite based on auto-restoration idea, i.e., continuous learning provide the ability to perform the detection, analyze, respond and classify failures without human intervention.

In the literature, there are a few approaches that propose methodologies to develop a system with continuous training to realize diagnosis. For example, in Pham and Cham (2007) is proposed an online learning asymmetric boosted classifiers for object detection. This approach is one of the first to try the continuous learning in techniques of pattern recognition. In the reference (Marchiori, Silveira, Lotufo, Minussi, \& Lopes, 2011) the authors presents a methodology to analyze the transient stability in electrical energy systems using, a Euclidean ARTMAP neural network with continuous training. In this paper the Euclidean ARTMAP neural network was modified to learning continuously and identify novelties in the analysis of the transient stability in electrical systems, i. e., the Euclidean ARTMAP neural network was able to identify unknown patterns, which were not learned yet in the training process. In Barros, Tonelli-Neto, Decanini, and Minussi (2015) the authors present a method to detection and classification of voltage disturbances in electrical power systems using a modified Euclidean ARTMAP neural network with Continuous Training. In this strategy the neural network is initially trained to obtain the knowledge. In the online process, if a pattern in analysis is unknown by the diagnostic system, is executed an online learning phase, and starting this, the diagnostic system is able to identify and classify the pattern that was unknown to the system. This method presents a precision of $90.32 \%$ in the voltage disturbance diagnosis.

In this sense, this work aims to propose a new approach to develop a system with continuous training to realize the voltage disturbances diagnosis in electrical distribution systems based on the Biological Immune System (BIS). Two immune algorithms were used to compose this system: the Negative Selection Algorithm (NSA) (Forrest, Perelson, Allen, \& Cherukuri, 1994) and the clonal selection algorithm (CLONALG) (de Castro \& Von Zuben, 2000).

The artificial immune algorithm, which is inspired by the BIS, is a promising technique in Intelligent Computing (IC). The BIS computationally reproduces the principal characteristics and the propriety functionalities and abilities. It has a stable, reliable, and adaptive architecture, allowing the continuous inclusion of the training module. This propriety allows the system to introduce new experiences and knowledge without the need to reinitialize the immune memory of the system. This is one advantage of using Artificial Immune Systems, which allow the possibility of continuous training unlike in other techniques such as using Artificial Neural Networks (ANNs). In some ANNs, to improve the training, it is necessary to reinitialize the process, consequently destroying the knowledge previously acquired, except for a few architectures that have the plasticity propriety as in the neural networks of the ART (Adaptive Resonance Theory) family (Carpenter, Grossberg, Markuzon, Reynold, \& Rosen, 1992). The main contribution and novelty of the approach proposed in this paper is the paradigm shift, that is, enable continuous learning for the pattern recognition methods. This ability allows great evolution to intelligent systems, where the methods are able to learn in online mode, acquiring knowledge of the unknown patterns for the system.

To evaluate and validate the efficiency of this new approach, the results were compared with a conventional diagnosis system based only in the NSA, proposed and presented in Lima, Lotufo, and Minussi (2013).

This paper is organized as follows. Section 2 discusses the BIS, Section 3 focuses on the NSA, and Section 4 tackles the CLONALG. The modeling and simulations is presented in Section 5 . The proposed methodology is presented in Section 6, and the applications and results are discussed in Section 7. The conclusions are presented in Section 8.

\section{Biological immune system}

The BIS is the principal defense of organisms against several infective agents that invade/infect the human body. In this case, the BIS must instantaneously act by effectively responding to the invading agents and identifying them to protect the human body (indicating the disease). There are two types of responses: the innate immune system and the adaptive immune system.

The innate immune system is the first defense line with a fast response characterized by dendritic cells (APC- Antigen Presenting Cell) and phagocyte (Granulocytes, Macrophages, etc.), which are responsible for ingesting strange particles to the organism and other types of defenses, such as physical barriers (skin) and chemicals (de Castro, 2001).

The adaptive immune system is the second level, which can recognize microorganisms, such as viruses, bacteria, fungi, protozoa, helminthes, and some types of worms. The adaptive immune system is responsible for realizing the learning process on the infective agents on the first contact with the antigens, i.e., the first exposure to the infective agent. In addition, it is responsible for creating memory immune cells from the first exposure to the infective agent to accelerate a response to this type of infective agent in future exposure (de Castro \& Timmis, 2002).

\subsection{Operation of the pattern recognition of the BIS}

The biological organism, particularly the human body, is composed of several cells and molecules that work in harmony and respond to strange agents that enter the organism, which are called antigens.

The BIS has fundamental characteristics such as pattern recognition, learning and neutralization of infective agents. These steps represent an immune response, which is illustrated on Fig. 1.

Step (I) represents the BIS exposure to an antigen (infective agent). The BIS can react with two types of responses: the innate immune response, which begins at step (II), and the adaptive immune response, which begins at step (VII). If the process begins with the innate immune system (step (II)), the infective agent is considered to be an unknown agent by the BIS, and the infective cell in the organism must be identified and informed a posteriori; then, the adaptive immune system begins an adaptive response (learn and generate antibodies to neutralize the antigen). If the process begins with the adaptive immune system (step (VII)) the antigen is known by the BIS, and the detection process is realized by the memory lymphocytes in the organism.

In step (II), the innate immune process begins when some antigen (infective agent) is ingested by a dendritic cell (APC- Antigen Presenting Cell). At this phase, the antigens are ingested and disintegrated into antigen peptides. In step (III), the pieces of peptides are linked to the MHC (Major Histocompatibility Complex) molecules and presented to the surface of the dendritic cell.

In step (IV), the $\mathrm{T}$ lymphocytes, which have receptive molecules at the surface, can recognize/identify different antigen $\mathrm{MCH} /$ peptides that are processed by the dendritic cells, i.e., when there is a link (combination), the antigen is recognized, 


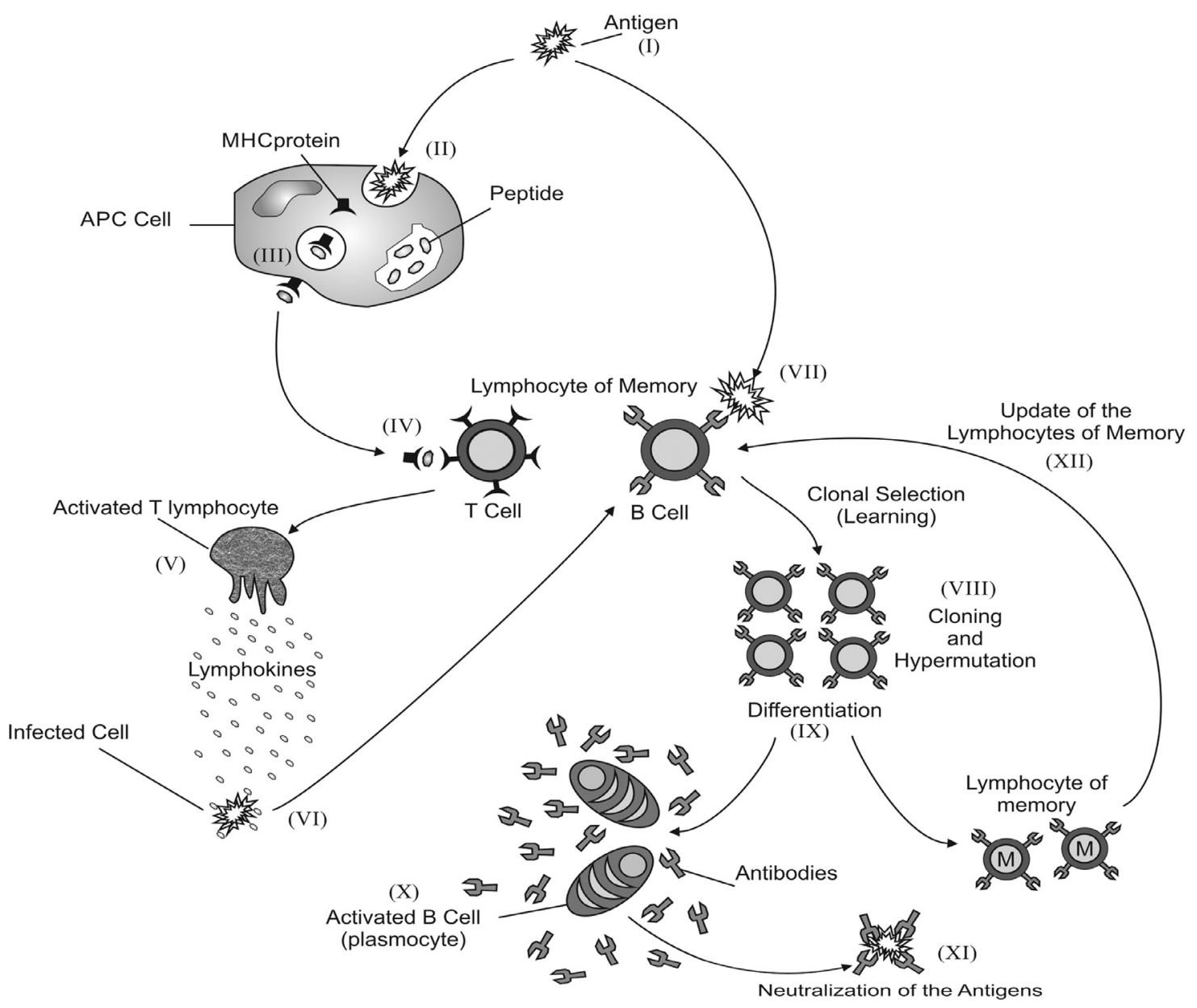

Fig. 1. Representation of the BIS.

and the lymphocyte state is activated. This step represents the proper/non-proper discrimination of the organism, which distinguishes proper cells from the infective agents (de Castro, 2001), (Dasgupta, 1998). Based on this pattern recognition principle, reference (Forrest et al., 1994) proposes the NSA. After the antigen detection, at step (V), the lymphocytes $\mathrm{T}$ are activated and secrete chemical signals (lymphokine) that suggest to other BIS components that a cell is infected.

After an infected cell is identified (step (VI)), B lymphocytes can recognize the infected cell and start the learning process, which is more commonly known as the principle of clonal selection (step (VII)). In this case, B lymphocytes can recognize free identified antigens without ingesting and digesting the presenting cells (APC).

When B cells recognize an antigen (by indication or memory), the clonal selection process begins, which is also known as learning process (step VIII). In this step, the lymphocytes pass under a clone and hyper-mutation process, which generates a set of B lymphocytes in the organism.

Then, step (IX) is a discerning process, where B lymphocytes with high affinity are separated to pertain to the memory set, and those with low affinity are activated and transformed in plasmocytes (step X) to secrete antibodies. In step (XI), the BIS-detected antigens are neutralized by the generated antibodies, which destroys the risk (disease).

In step (XII), the high-affinity lymphocytes in the distinguishing process are transformed and pertained to memory lymphocytes to substitute for those with low affinity. The new memory lymphocytes begin to circulate in the organism to ensure an efficient and fast response to a future exposure to the same antigen (infective agent).

It is emphasized that the entire process is realized with the cooperation of the cells that form the BIS, each of which is responsible for a relatively simple function, and the set that realizes an extremely complex work (de Castro, 2001), (Dasgupta, 1998).

This paper was inspired by the BIS operation, pattern recognition and learning processes.

\section{Negative selection algorithm}

The NSA is based on the pattern recognition process of the biological immune system and elaborated as a computational model. The NSA is proposed in Forrest et al. (1994). In this algorithm (negative selection) the $\mathrm{T}$ lymphocytes that occur at a specific time and discriminates the proper/non-proper cells in the organism. The algorithm is executed in two phases, such as presented in Figs. 2 and 3 (de Castro, 2001):

The censor phase of the NSA consists primarily of generating a detector set from the data that were randomly chosen and verifying which data can recognize a non-proper pattern. The detectors are similar to the mature $\mathrm{T}$ cells that can recognise pathogenic agents (Lima et al., 2013).

The monitoring phase consists of monitoring a system to identify a change in behaviour; thus, this monitoring phase classifies the change using the detector set that was created in the censor phase. The censor phase occurs in the off-line mode, and the monitoring phase occurs in real-time mode (de Castro \& Timmis, 2002), (Dasgupta, 1998). 


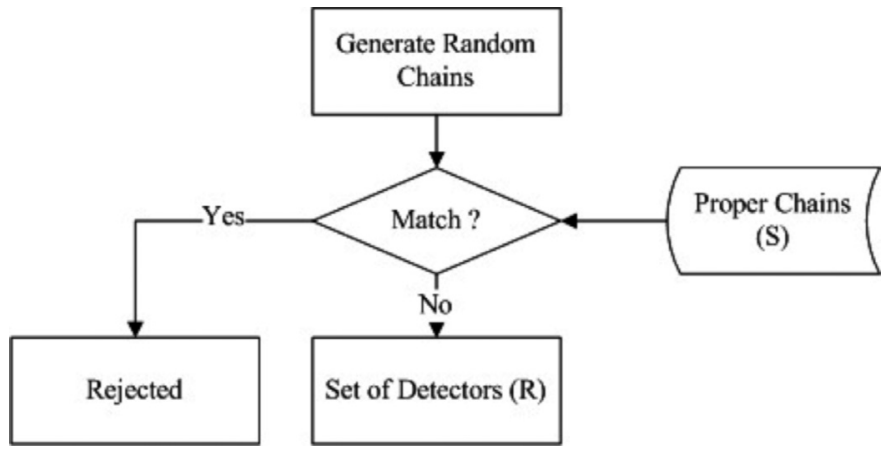

Fig. 2. Flowchart of the censor phase of the NSA.

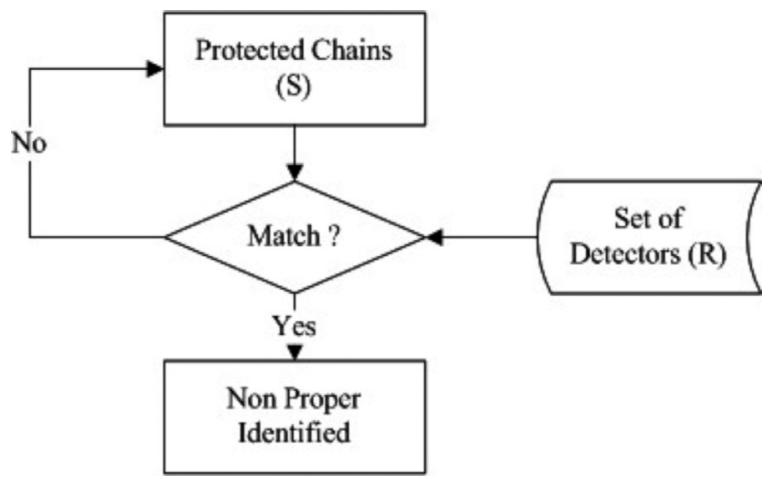

Fig. 3. Flowchart of the monitoring phase of the NSA.

The chains at the NSA represent the patterns by data structures (vectors). These chains can be classified in two types of NSA: the antigens $(A g)$ and the lymphocytes $(A b)$. The $A g$ is the signal to be analyzed, unknown by the NSA and represented by expression (1). The detectors, that is, the lymphocytes $(A b)$, are expressed according to expression (2) (Lima et al., 2013), (de Castro \& Timmis, 2002):

$A g=A g_{1}, A g_{2}, A g_{3}, \ldots, A g_{L}$

$A b=A b_{1}, A b_{2}, A b_{3}, \ldots, A b_{L}$

where:

$L$ is the dimension of the lymphocytes $(A b)$ and antigens $(A g)$.

It is emphasized that the censor and monitoring phases of the NSA are executed off-line and on-line, respectively (de Castro \& Timmis, 2002).

\subsection{Matching criterion and affinity rate}

A criterion called matching or combination is used to evaluate the affinity with the chains (antigens and lymphocytes) and determine their similarity. The matching can be perfect or partial.

This paper uses the partial matching and affinity proposed in Bradley and Tyrrell (2002). The affinity rate represents the similarity grade with the two analyzed chains. According to Bradley and Tyrrell (2002), the affinity is defined as follows:

$T A f=\left(\frac{A n}{A t}\right) 100$

where:

TAf affinity rate;

An number of normal chains in the problem (proper chains); At total number of chains in the problem (proper and nonproper chains).
The precise affinity rate can be calculated using Eq. (3), where a statistical relation with the samples is proposed.

A deviation, i.e., a tolerance rate, is proposed where the combination with the patterns is acceptable and can improve the diagnosis (Lima et al., 2013):

$\underline{A b}_{i} \leq A g_{i} \leq \overline{A b}_{i}$

where:

$\mathrm{Ag}_{i} \quad$ nominal value of position $i$ of the antigen (pattern under analysis);

$\underline{A b}_{i} \quad$ nominal value of position $i$ minus the deviation adopted in the antibody (detector pattern);

$\overline{A b}_{i} \quad$ nominal value of position $i$ plus the deviation adopted in the antibody (detector pattern).

Expression (5) represents the method to quantify the total affinity with the analyzed patterns (Bradley \& Tyrrell, 2002), (Lima \& Minussi, January-2014):

$A f_{T}=\frac{\sum_{i=1}^{L} P C}{L} 100$

where:

$A f_{T} \quad$ percentage of affinity with the analyzed patterns;

$L \quad$ total number of positions;

PC matching position;

$\sum_{i=1}^{L} P c \quad$ sum (number) of the matching positions.

Thus, if $A f_{T}$ is greater than $T A f$, then the combination/matching with the patterns occurs, i.e., the patterns are considered equal/similar. Otherwise, there is no matching with the patterns.

\section{Clonal selection algorithm}

The clonal selection algorithm, CLONALG, was originally proposed in de Castro and Von Zuben (2000). There are two available versions of the algorithm in the literature, one of which solves machine learning problems and pattern recognition, and the other solves optimization problems (de Castro, 2001).

The CLONALG for recognition problems and machine learning has the following steps (de Castro, 2001), (de Castro \& Timmis, 2002):

Step I: Initialization: generate a random population $\left(A b=A b_{\{M\}}+\right.$ $\left.A b_{\{R\}}\right)$ with $N$ lymphocytes for each antigen (Agi). $N$ is given by $M+R$;

Step II: Affinity evaluation: each antigen $(A g i)$ is presented to every $A b$ of the population $(A b)$ in a process of affinity evaluation. A vector $f$ of affinity is determined;

Step III: Selection: the $n$ Abs with high affinity $f$ to Agi are selected to compose a sub population $\left(A b_{\{n\}}\right)$;

Step IV: Cloning: the $n$ selected $A b$ s proliferate (clone) proportionally to the affinities of the antigen (Agi) and generate a $C$ population of clones in each selected $A b$;

Step V: Hyper-mutation: the $C$ population of clones is submitted to the maturation affinity process, which generates a new population, $C^{*}$, where each $A b$ will mutate with a rate inversely proportional to the affinity $f$;

Step VI: Affinity evaluation: determine the affinity $f^{*}$ with the set $C^{*}$ of mutated clones and the antigen Agi;

Step VII: Re-selection: from the mature population $C^{*}$, re-select the $n$ best mature $A b$ s to make a sub-population $\left(A b_{\{n\}}\right)$. From this sub-population, choose the best $A b s$ to enter the memory set $\left(A b_{\{n\}}\right)$. A $A b$ enters the memory set if it has high affinity rate and can substitute a $A b$ of the memory; 
Step VIII: Meta-dynamics: substitute $d$ antibodies of $\left(A b_{\{R\}}\right)$ by new individuals $\left(A b_{\{d\}}\right)$ to make the repertoire diverse. The anti-bodies with the lowest affinity are substituted; Step IX: Repeat steps II-VIII until the stop criterion is satisfied.

At the end of the iterative process, the memory set $\left(A b_{\{M\}}\right)$ has $M A b s$ with high affinity rates to the antigen (Agi). This memory set can be used by the NSA to detect and classify learned antigens in the clonal selection process.

For learning problems, step VIII (meta-dynamics) is not executed; therefore, $d=0$.

The number $N_{c}$ of clones generated in Step IV for each $A b i$ is given by Eq. (6) (de Castro, 2001):

$N_{c}^{i}=\operatorname{round}\left(\frac{\beta N}{i}\right)$

where:

$\beta$ is a multiplicative factor between [0,1], $N$ is the total number of $A b s$ of population $A b$, and round(.) is the approximation operator to the closest integer.

The mutation rate $(\alpha)$ of each clone is defined according to Eq. (7) (de Castro, 2001):

$\alpha=\exp \left(-\rho f^{n}\right)$

where:

$\rho$ is a control damping parameter of the exponential function and $f^{n}$ is the normalized value of affinity $f$, which is calculated using Eq. (8).

$$
f^{n}=\frac{f}{f_{\max }}
$$

Then, each clone mutates according to (9) (de França, Von Zuben, \& de Castro, 2005):

$m=\operatorname{round}\{\alpha N(0,1)\}$

where:

$m$ is the number of mutations that each clone has, round(.) is the approximation operator to the closest integer, $\alpha$ is the mutation rate, and $N(0,1)$ is the Gaussian random variable with mean zero and $\sigma=1$.

\section{Modelling and disturbance simulation}

Although there was no database of distribution systems under perturbations, the data to evaluate the methodology was provided simulating events in test systems.

Using the routine models (Dubé, 1996) from the EMTP software (EMTP-RV 2011), a real distribution system with 134 buses was modeled. A sample frequency of $15.36 \mathrm{kHz}$, which is equivalent to 256 samples per cycle, was used as a parameter. The simulation time was $200 \mathrm{~ms}$.

For the voltage disturbances, the theoretical model proposed by Abdel-Galil, Kamel, Youssef, El-Saadany, and Salama (2004) was used for the simulations, where each disturbance was generated by varying the parameters in the equations that represented the waveform of the disturbances.

In total, 624 simulations were executed for the 134-bus system (LaPSEE 2011). The simulations considered the phase where the disturbance occurred, 50-120\% to loading system, and the parameters of the theoretical model. Table 1 presents the quantity of executed simulations for each class of disturbance.
Table 1

\begin{tabular}{lc} 
Number of simulations executed. \\
\hline Voltage disturbance & 134 buses \\
\hline Outage & 48 \\
Harmonic & 144 \\
Swell & 72 \\
Sag & 72 \\
Swell with harmonic & 96 \\
Sag with harmonic & 96 \\
Oscillatory transient & 96 \\
Total & 624
\end{tabular}

\section{Proposed methodology}

This section describes the methodology proposed to realize the voltage disturbance diagnosis in electrical distribution systems using the artificial immune algorithm with continuous-learning.

The voltage disturbance diagnosis system with continuouslearning in this section is inspired by the pattern recognition and learning process of the BIS, which was described in Section 2.A of this work. To computationally formulate and reproduce this biological process, the NSA proposed by Forrest et al. (1994) and the CLONALG proposed by de Castro and Von Zuben (2000) were used. The NSA realizes the voltage diagnosis disturbance and the CLONALG as a strategy for learning.

The diagnosis system with continuous-learning has five principal steps: NSA (off-line) censoring, novelty detection, continuouslearning, NSA monitoring and knowledge updating. The block diagram is shown in Fig. 4. According to Fig. 4, in the off-line process called censor module, the proper and disturbance detectors for the NSA are defined, which form the set of NSA detectors.

Thus, from the data acquisition system (SCADA (Wylie \& Pleydell, 2008)), voltage readings are executed at the electrical distribution substation to obtain the oscillographs. Then, the censor module defines the proper detector set. For electrical distribution systems, the proper signals have the normal operation characteristics. Then, 256 window samples of the signal that represents the normal system operation are filed as proper detectors.

Subsequently, the censor module is executed to generate the disturbance detectors, and for each type of disturbance, the censor module is executed once.

In this phase, the detectors are randomly generated from a set of signals that represent the disturbed system. Thus, 1 cycle (256 points) in the simulated data is windowed and these data are compared window by window with the proper detectors. If there is matching, the random vector is rejected. Otherwise, it is accepted and filed as a disturbance detector in the set of the disturbance detectors. The censor process is illustrated in Fig. 5.

The system operator defines the number of detectors (proper or disturbances). The detector set is used for the novelty detection and monitoring steps of the NSA.

The monitoring process (on-line) is executed after the off-line process. The test set (antigens), which is obtained from the acquisition system (SCADA (Wylie \& Pleydell, 2008)), is formed using every available signal in the database. Then, a random signal is selected to be analyzed.

The novelty detection step is executed when a signal is selected to be analyzed, and the analyzed signal is compared with the detector set of the NSA. The matching of the signal with some detector is verified. If they match, the analyzed signal is known by the system, i.e., there is no novelty. Otherwise, the signal is considered a novelty or unknown by the system.

When the system identifies a novelty, the continuous-learning step is executed to learn about the new antigen (analyzed signal), and the learning process occurs, which is realized by the CLONALG. 


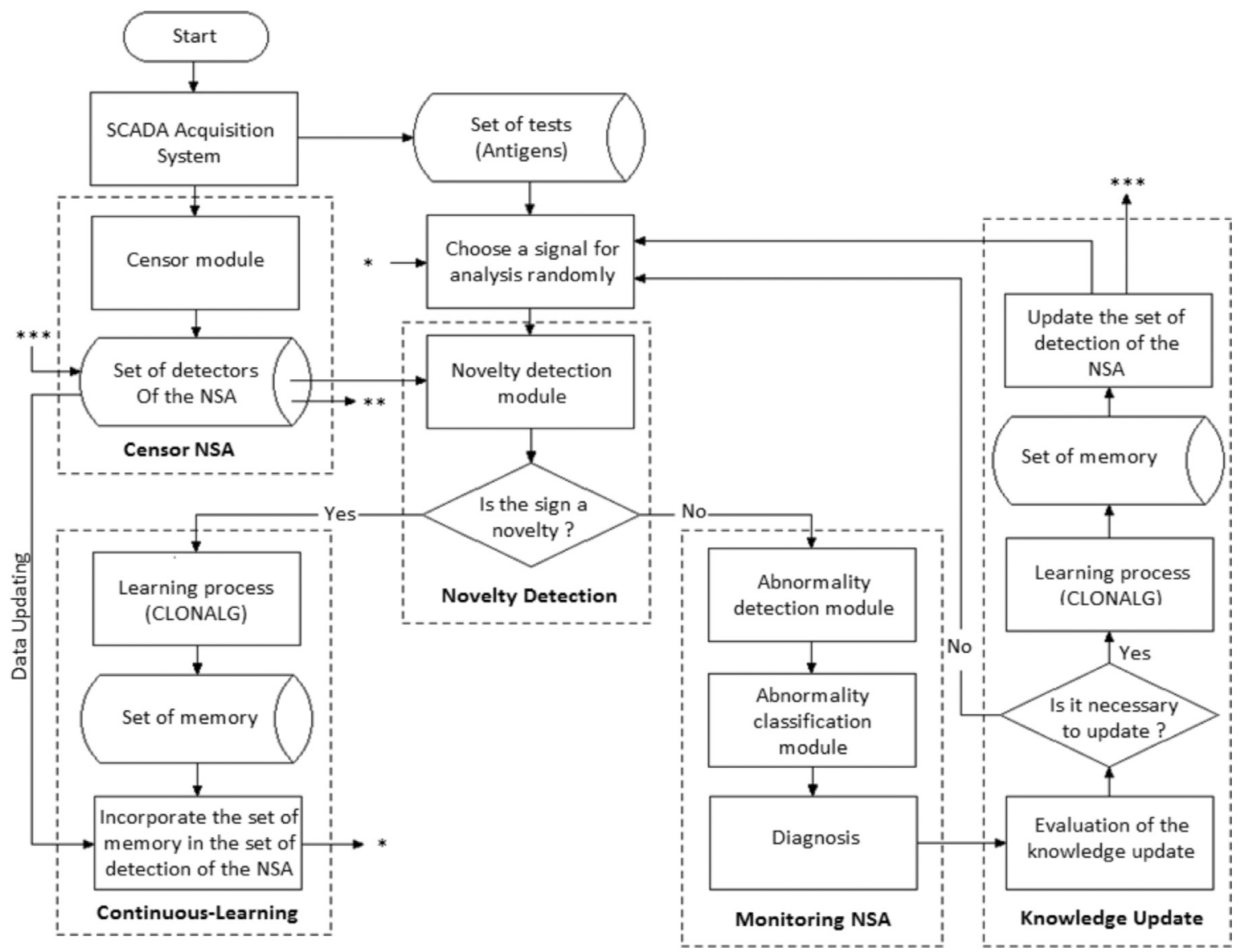

Fig. 4. Diagnosis system with continuous-learning.

The objective of the CLONALG is to generate a memory set (knowledge) from an unknown signal (antigen). This process follows the steps in Section 4 of this work. Initially, a population of lymphocytes is randomly generated. To randomly generate a lymphocyte, the maximum and minimum values of the voltage signal to be detected (antigen) are identified, and a vector with 256 positions is created and randomly filled with real values between the maximum and minimum voltages, which generates a random signal.

Eq. (5) is used to quantify the affinity with the lymphocytes of the population $(A b)$ and the antigen. Then, the $n$ best lymphocytes with highest affinity values to the antigen are selected for the clonal process.

To quantify the affinity with the lymphocytes of the population $(A b)$ and the antigen, Eq. (5) is used. Then, the $\boldsymbol{n}$ best lymphocytes with highest affinity values to the antigen are selected for the cloning and hyper-mutation processes. The number of clones is calculated using Eq. (6), and the number of mutations to be realized on the clones depends on the calculus of Eqs. (7)-(9). Mutation is an evolutionary process to execute little modifications in the lymphocyte structure to increase the affinity to the antigen.

Due to the analyzed signals are expressed by vectors with real positive and negative numbers, this method uses the inductive mutation techniques (Wylie \& Shakhnovich, 2012), which is an evolutionary-algorithm technique to solve this type of problems. Thus, the mutation process consists of realizing a mutation using Eq. (10) in a random position of the signal (lymphocyte).

$A b_{i}^{\prime}=\left\{\begin{array}{l}A b_{i}+\alpha^{*}\left(A b_{i}-A g_{i}\right), A b_{i}>0 \\ A b_{i}+\alpha^{*}\left(A g_{i}-A b_{i}\right), A b_{i}<0\end{array}\right.$ where: $A b_{i}^{\prime}$ is the mature lymphocyte, $A b_{i}$ is the position to be mutated, $A g_{i}$ is the goal (learning pattern), and $\alpha$ is a random number between $[0,1]$.

After the lymphocytes are matured, the $n$ best matured clones are re-selected to be included in the population. The selected lymphocytes replace the worst lymphocytes of the population. Furthermore, the best lymphocytes are separated for the memory set. The process is repeated until the stop criterion is satisfied. In this work, the stop criterion is that every lymphocyte of the memory set reaches at least $92 \%$ affinity with the antigen (signal to be learned).

Fig. 6 illustrates the learning process of CLONALG to learn a harmonic signal. Fig. 6(a) illustrates the signal to be learned. Fig. 6(b) shows the memory antibody randomly generated in red in the iteration 1. Fig. 6(c), (d), (e), (f), (g), (h) and (i) shows the memory anti-body after 50,100,150, 200, 250, 300 and 350 iterations, respectively. Finally, Fig. 6(j) illustrates the memory anti-body after the stop criterion is satisfied, i.e., the affinity with the random signal and the pattern to be learned are greater than $92 \%$.

At the end of the CLONALG process, there is a memory set (knowledge) related to the unknown antigen. This knowledge set is included to the detector set of the NSA to provide knowledge to the system. For example, in another analysis of the same type, the system can recognize and classify the disturbance. This characteristic is known as continuous-learning.

When the novelty detection step does not identify a novelty, i.e., when the antigen is known by the NSA, the process occurs according to Fig. 7, similarly to the conventional NSA. Thus, the signal under analysis must be compared with the detector set of the NSA and evaluate the matching and affinity to detect the abnormality and later classify the signal according to the class of disturbance where matching occurs. 

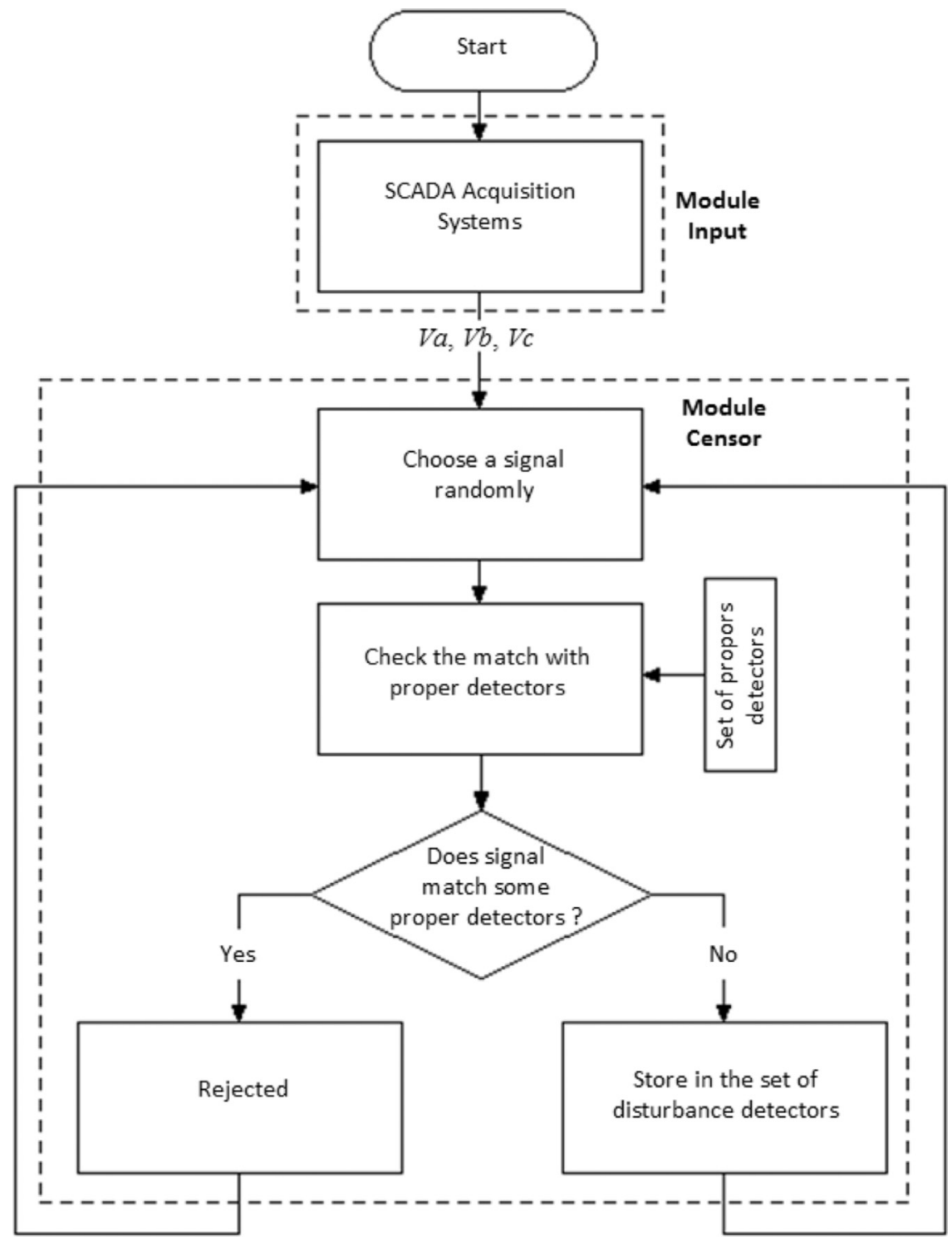

Fig. 5. Censor Module of NSA.

After the signal is analyzed, the monitoring process of the NSA finishes.

Furthermore, the knowledge module is updated, which is responsible for evaluating whether there is a requirement to update the detector set of the NSA (knowledge) to improve the disturbance detection and classification processes. This case uses the matching of the detectors with the antigen signal (signal under analysis) as the criterion. If $70 \%$ of the disturbance detectors match the analyzed signal, it is not necessary to update the detection set of the NSA for the signal classified. If less than $70 \%$ of the detectors do not match with the classified pattern, the knowledge-updating process occurs. When the NSA cannot recognize the signal with less than $70 \%$ of the detectors, it is necessary to update the detector set of the NSA to improve the recognition process and use the antigen to learn (obtain knowledge).

Therefore, it is necessary to update the knowledge of the CLONALG learning process, which is executed according to the previous description and identical to the process realized by the continuouslearning module.

After the learning process and a set of memory lymphocytes of the antigen signal (signal under learning) is obtained, the best lymphocyte of the memory set must be selected and updated to the detector set of the NSA. The updating occurs on the substitution of one detector that does not match the antigen pattern. This detector is substituted by the best lymphocyte of the memory set generated on the learning process realized by the CLONALG.

The learning updating only occurs when there is the need to reinforce the NSA knowledge, i.e., when fewer than $70 \%$ of the detectors do not satisfy the affinity criterion, matching no antigen. Then, this detector is substituted by a new detector generated in the learning process, which is called the updating knowledge.

The process in Fig. 4 is repeated until every antigen (test set) is analyzed.

\section{Results and applications}

This section presents the obtained results of the proposed method in the simulated test system. The algorithm was developed using MATLAB ${ }^{\circ}$ (MATLAB 2011). Every test and simulation was performed using a PC Intel Core 2 Duo $1.9 \mathrm{GHz}$ with $2 \mathrm{~GB}$ of RAM (Random-Access Memory). The proposed algorithm used a real distribution system with 134 buses (LaPSEE 2011). 
a
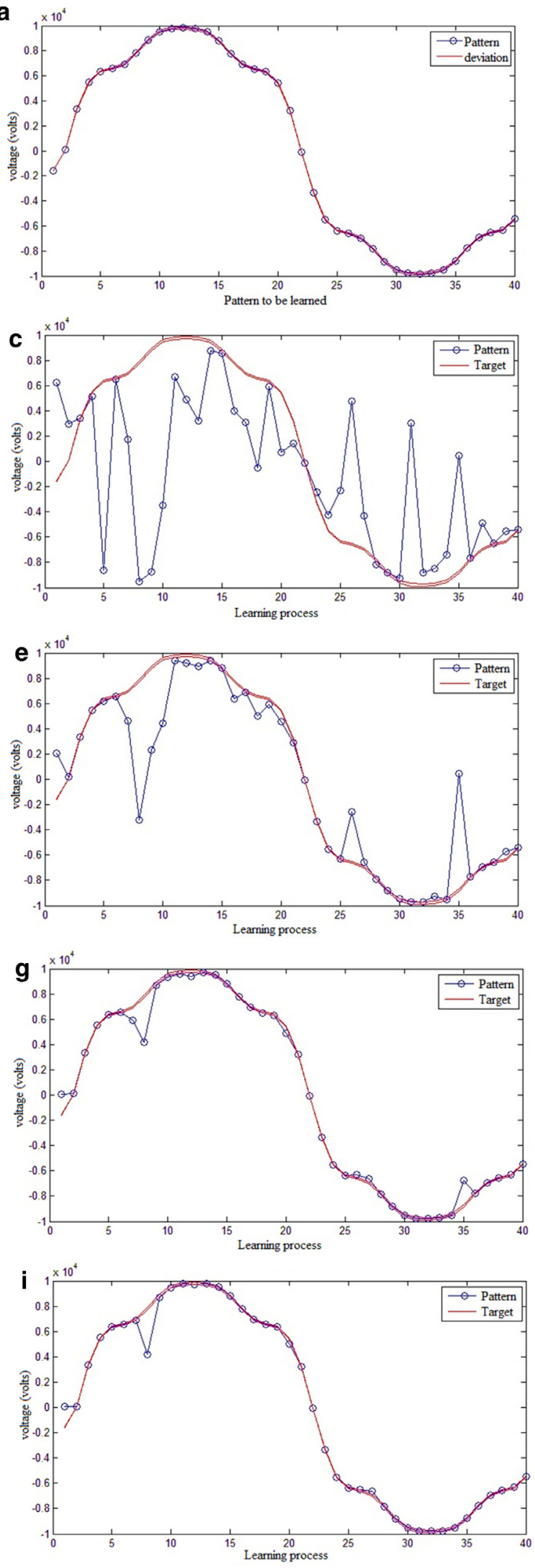
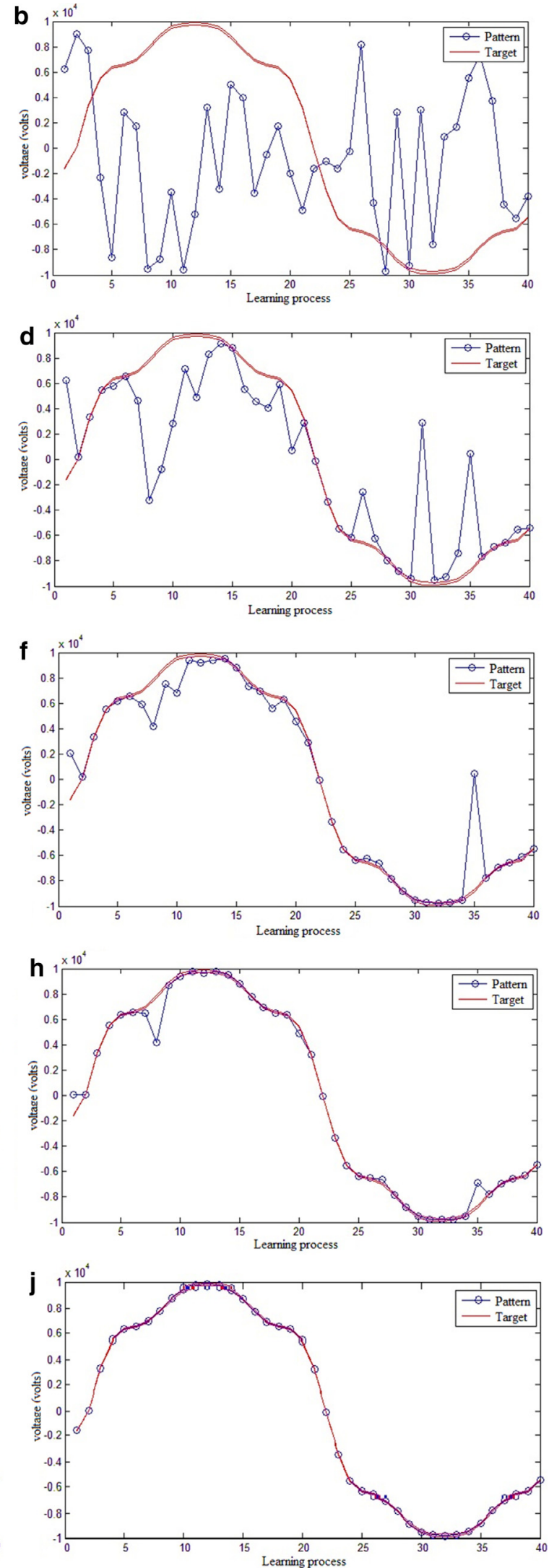

Fig. 6. Learning process of the CLONALG. 


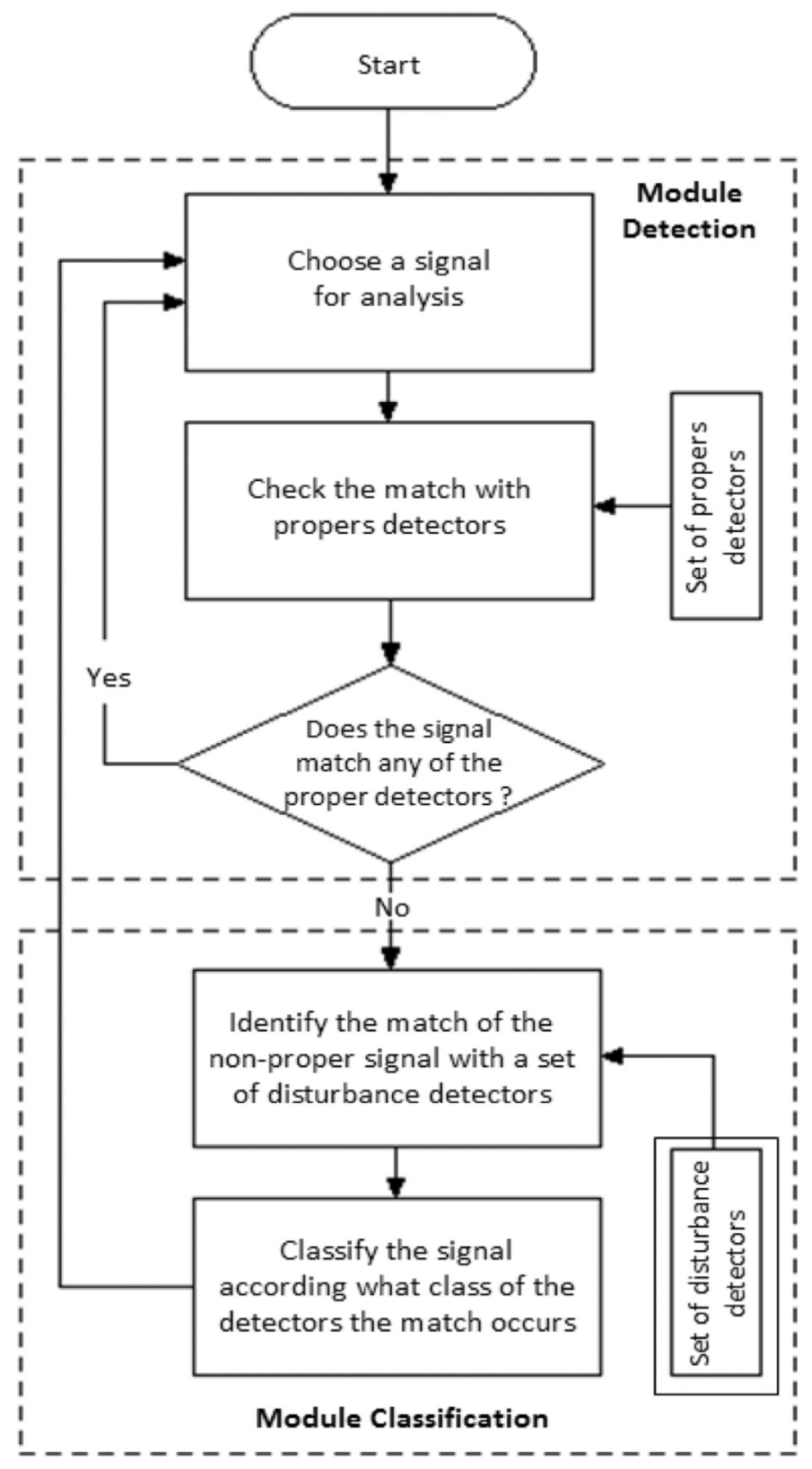

Fig. 7. Monitoring Module of the NSA.

The proposed method was evaluated to verify the efficiency, precision and robustness in the voltage disturbance diagnosis using the conventional algorithm (without continuous training), proposed and explained in Lima et al. (2013), and the algorithm with continuous training. The metrics adopted to the efficiency, precision and robustness are: ability to learn unknown patterns, percentage of correct answers and computational time.

Table 2 presents the parameters in the tests, which were obtained using an empirical test process.

Three patterns were excluded during the censor phase in both systems (with and without continuous training) to evaluate the performance of the novelty-detection, continuous-learning and knowledge-updating modules of the proposed algorithm. The excluded patterns were: swell, harmonic and oscillatory transient. They were randomly chosen like any disturbance, and the algorithms began the monitoring process online without knowing these patterns.
Table 2

Parameters.

\begin{tabular}{lll}
\hline Parameters & NSA without continuous training & NSA with continuous training \\
\hline$T A f$ & $66.66 \%$ & $66.66 \%$ \\
$N$ & - & 25 \\
$M$ & - & 9 \\
$R$ & - & 16 \\
$n$ & - & 5 \\
$\beta$ & - & 0.3 \\
$\rho$ & - & 4 \\
$d$ & - & 0 \\
\hline
\end{tabular}

Table 3

Results of the system without continuous training.

\begin{tabular}{lccc}
\hline Disturbances & \multicolumn{2}{l}{134 buses } & \\
\cline { 2 - 4 } & Tested patterns & Matching patterns & Matching (\%) \\
\hline Swell & 72 & 0 & 0.00 \\
Sag & 72 & 70 & 97.22 \\
Outage & 48 & 48 & 100.00 \\
Harmonic & 144 & 0 & 0.00 \\
Swell with harmonic & 96 & 96 & 100.00 \\
Sag with harmonic & 96 & 95 & 98.95 \\
Oscillatory transient & 96 & 0 & 0.00 \\
Total & 624 & 309 & 49.51 \\
\hline
\end{tabular}

Table 4

Results of the continuous-training system.

\begin{tabular}{lccc}
\hline Disturbances & \multicolumn{2}{l}{134 buses } & \\
\cline { 2 - 4 } & Tested patterns & Matching patterns & Matching (\%) \\
\hline Swell & 72 & 71 & 98.61 \\
Sag & 72 & 72 & 100.00 \\
Outage & 48 & 48 & 100.00 \\
Harmonic & 144 & 140 & 97.22 \\
Swell with harmonic & 96 & 96 & 100.00 \\
Sag with harmonic & 96 & 96 & 100.00 \\
Oscillatory transient & 96 & 95 & 98.95 \\
Total & 624 & 618 & 99.03 \\
\hline
\end{tabular}

Table 3 shows the results with the previously mentioned patterns and configurations for the conventional diagnosis, i.e., the diagnosis without continuous training.

The conventional diagnosis system cannot identify the excluded patterns from the censor process, i.e., it cannot identify these types of patterns.

Table 4 presents the results of the diagnosis process with continuous training. The configurations and parameters are identical to those used for the conventional NSA algorithm.

Table 4 shows that the continuous-training system can learn the unknown disturbances and execute the diagnosis in another analysis. In addition, the updating knowledge module contributes to increase the matching rate for some known disturbances because the system updates the detector set of the NSA in the online monitoring process, which reinforces and improves the knowledge.

Tables 3 and 4 show an improvement in diagnosing the disturbances, i.e., the system with continuous training can evolve with the experience and diagnose the disturbances that were not previously diagnosed. The excluded patterns from the censor process were not recognized by the system without continuous training with $0 \%$ matches. After using the continuous training, the patterns are identified with a matching rate of approximately $98.95 \%$ for the swell, $97.22 \%$ for the harmonic and $98.95 \%$ for the oscillatory transient.

In conclusion, it is possible to control the quality of the generated lymphocytes in the memory set of the learning process with the CLONALG. To finish the learning process, the lymphocytes of 


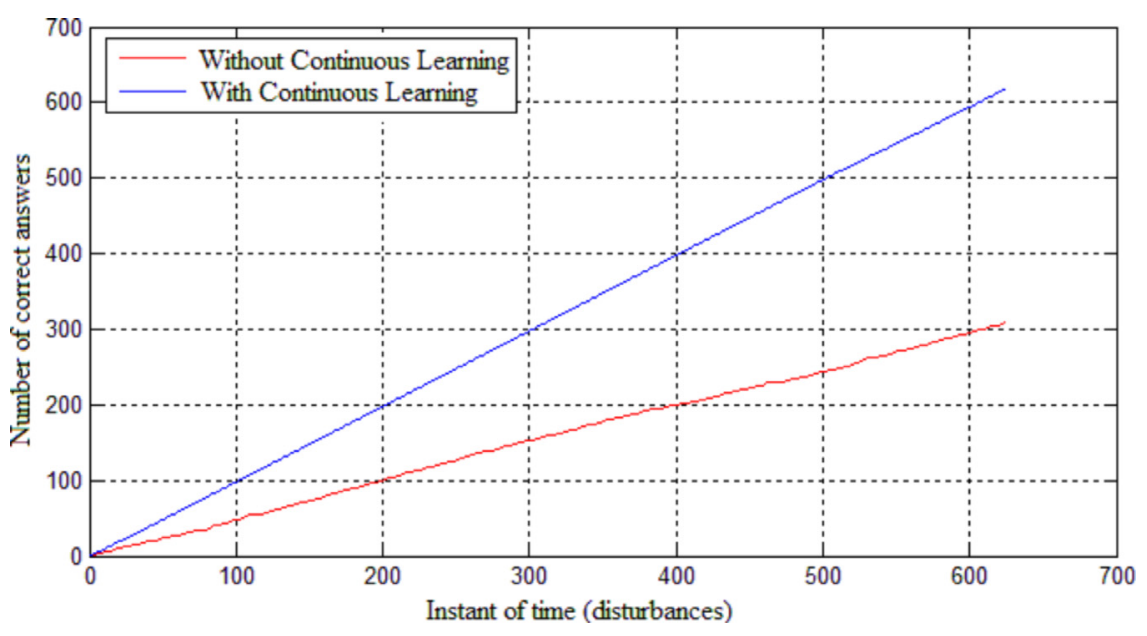

Fig. 8. Graph of the efficiency with time.

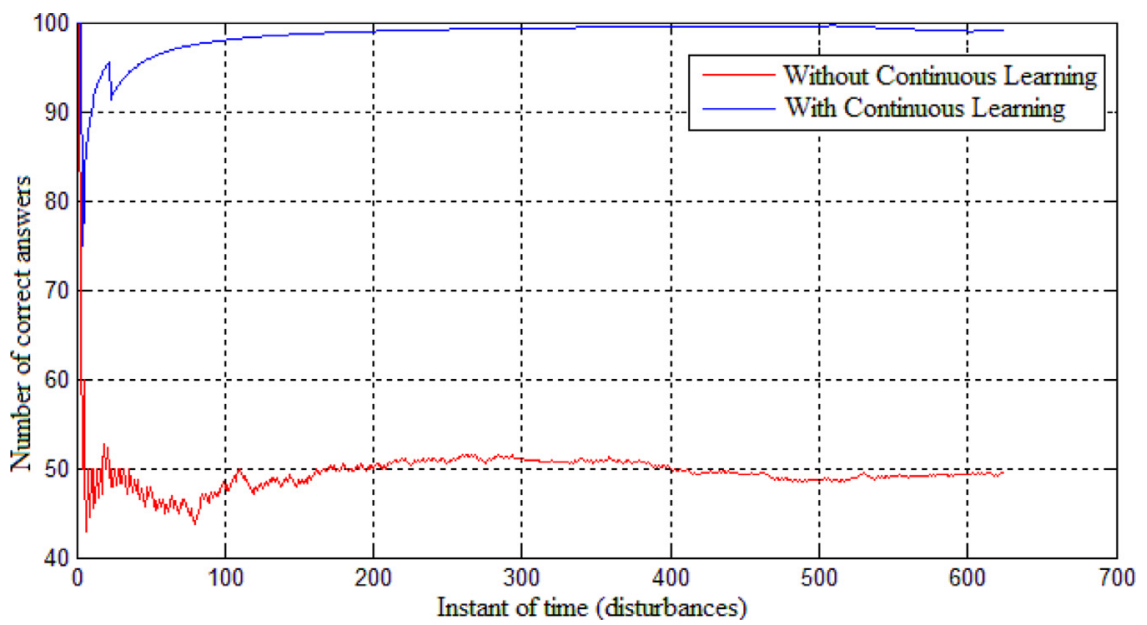

Fig. 9. Graph of the precision with time.

the memory set and the antigens (pattern to be learned) must have an affinity of at least $92 \%$. With this criterion, it is possible to ensure that the memory lymphocytes have high affinity values and provide efficiency in the diagnosis process.

Figs. 8 and 9 show graphs of the obtained results with and without continuous training to analyze the matches and the precision in a given time. The instant of time is the occurrence of a failure, i.e., the quantity of failures that the system analyzes until the considered instant.

Fig. 8 shows a linear relationship between the instant of time (number of failures) and the number of matches until this instant of time. When the system continuously learns, the linear relationship tends to have an angular coefficient of approximately 1 and a linear coefficient of approximately 0 for each analyzed instant, i.e., an inclination of approximately $45^{\circ}$ represents an optimal precision. For the system without continuous training, the linear relationship presents different coefficients with an inclination of approximately $22^{\circ}$, which confirms that after the continuous training, the system improves the performance with time.

Finally, Fig. 9 shows the precision of the system considering the analyzed instants of time (failures) until the moment. The system without continuous training has a low error precision of approximately $50 \%$ until the end. The system with continuous training has almost no error and maintains a precision of approximately $100 \%$ during the entire time.

\subsection{Comparative study}

Table 5 presents a comparative study that uses matching according to the proposed method and the principal references that are available in the literature. This study presented methods with continuous learning and methods without continuous learning.

The proposed method presents a matching index and a precision that is similar to other works that are available in the literature. It should be emphasised that the use of the continuous learning changes the sensitivity of the voltage disturbance diagnosis and can detect and classify any abnormality; therefore, good results are presented.

\subsection{Comments about the algorithm}

After carrying out the tests and obtaining the results, we identified the strengths and weaknesses of the proposed algorithm; these are presented in this section.

Strengths:

- The proposed algorithm presented excellent performance, presenting a good accuracy rate (99.03\%) when compared to the best works available in the literature.

- The algorithm require low processing time.

- This algorithm has the capacity to identify unknown patterns (unknown disturbances). 
Table 5

Comparative Study.

\begin{tabular}{|c|c|c|c|}
\hline Reference & Electrical system/theoretical model & Method used & Total match (\%) \\
\hline (Uyar et al., 2008) & (Abdel-Galil et al., 2004) & Multilayer Perceptron (Levenberg-Marquardt) e wavelet & 96.21 \\
\hline (Zhang et al., 2011) & Not specified & Decision tree and Fourier transform & 99.00 \\
\hline (Lima et al., 2013) & 134-bus & Negative Selection Algorithm & 99.11 \\
\hline (Oleskovicz et al., 2009) & $138 / 13,8 \mathrm{kV}$ & Multilayer Perceptron e wavelet & 99.31 \\
\hline $\begin{array}{l}\text { (Decanini, Tonelli-Neto, Malange, \& } \\
\text { Minussi, 2011) }\end{array}$ & 134-buss & Fuzzy ARTMAP neural network and wavelet transform & 99.66 \\
\hline (Lima, Lotufo, \& Minussi, 2015) & 134-buss & Negative Selection Algorithm with wavelet transform & 100.00 \\
\hline (Lima et al., 2015) & 134-buss & Modified Negative Selection Algorithm & 100.00 \\
\hline (Barros et al., 2015) & 134-bus & $\begin{array}{l}\text { Modified Euclidean ARTMAP neural network with } \\
\text { continuous learning }\end{array}$ & 90.32 \\
\hline This work & 134-bus & Immunologic algorithm with continuous learning & 99.03 \\
\hline
\end{tabular}

- This method has the capacity to continuously improve/reinforce the knowledge and permanently learn without reinitializing the learning process.

- This method is able to follow innovations and modernizations of the electrical system, which include new types of prominent failures, learn with experience and constantly evolve.

Weaknesses:

- The algorithm proposed have several parameters that must be set in advance.

\section{Conclusion}

This work presents a new approach for voltage disturbance diagnosis with continuous training based on the BIS. Two artificial immune algorithms were used for the diagnosis and learning: NSA and CLONALG. The continuous learning algorithm is compared with the conventional algorithm, i.e., without continuous learning, proposed and explained in Lima et al. (2013). Three patterns were excluded from the censor process of both systems to evaluate the novelty detection, and the conventional process matches $49.51 \%$ of the analyzed disturbances. The algorithm with continuous learning matches $99.03 \%$ for every pattern.

The continuous-learning module can continuously learn to identify unknown disturbances. The obtained results show that the diagnosis with continuous learning performs better (in terms of efficiency, precision and robustness) than the system without the continuous learning. Therefore, the newly proposed detection strategies in this paper, which continuously learn and update knowledge, provide the diagnosis system with sufficient precision, efficiency and robustness for important applications and the capacity to follow the evolution of electrical systems such as the smartgrid environment. The main advantage of this method is the capacity to continuous learning, which is a novelty in methods of voltage disturbances diagnosis. This advantage is very important to evolution of the techniques in intelligent systems.

The results allow practical applications such as modeling and development of an efficient system for intelligent diagnosis in smart grids. Based on the accuracy index of the computational algorithm, certainly in a real network with modern relays or a robust system of acquisition data, analysis and diagnosis should provide better efficiency.

Finally, the proposed methodology when compared to other methods such as artificial neural networks presents the capacity to continuously improve/reinforce the knowledge and permanently learn without reinitializing the learning process, improving the diagnosis process.

To give sequence to this research, it is suggested the following topics: add module to perform wavelet analysis and processing of signals; investigate the possibility of investing in distribution systems environment in anticipatory character; apply the method proposed to distribution systems with non-linear loads and distributed generation.

\section{References}

Abdel-Galil, T. K., Kamel, M., Youssef, A. M. E., El-Saadany, F., \& Salama, M. M. A. (2004). Power quality disturbance classification using inductive inference approach. IEEE Transactions on Power Delivery, Piscataway, 19(4), 1812-1818.

Alag, S., Agogino, A. M., \& Morjaria, M. (2001). A methodology for intelligent sensor measurement, validation, fusion, and fault detection for equipment monitoring and diagnosis. Artificial Intelligence for Engineering Design, Analysis and Manufacturing, Cambridge, 15(4), 307-320.

Barros, A. C., Tonelli-Neto, M. S., Decanini, J. G. M. S., \& Minussi, C. R. (2015). Detection and classification of voltage disturbances in electrical power systems using a modified euclidean ARTMAP neural network with continuous training. Electric Power Components and Systems, 1, 1-11.

Bradley, D. W., \& Tyrrell, A. M. (2002). Immunotronics - novel finite-state-machine architectures with built-in self-test using self-nonself differentiation. IEEE Transactions on Evolutionary Computation, 6, 227-238.

Carpenter, G. A., Grossberg, S., Markuzon, N., Reynold, J. H., \& Rosen, D. B. (1992). Fuzzy ARTMAP: a neural network for incremental supervised learning of analogy multidimensional maps. IEEE Transactions on Neural Network, 3, 689-713.

Dasgupta, D. (1998). Artficial immune systems and their applications. Secaucus, NJ: Springer-Verlag New York, Inc.

de Castro, L. N. (2001). Immune engineering: Development and application of computational tools inspired by artificial immune systems. Ph.D. thesis. Brazil: UNICAMP, Campinas (SP) (in Portuguese).

de Castro, L. N., \& Timmis, J. (2002). Artificial immune systems: a new computational intelligence approach (1st edition). Springer.

de Castro, L. N., \& Von Zuben, F. J. (2000). The clonal selection algorithm with engineering applications. In Proceedings of GECCO, workshop on artificial immune systems and their applications, Las Vegas (pp. 36-39).

de Franca, F. O., Von Zuben, F. J., \& de Castro, L. N. (2005). An artificial immune network for multimodal function optimization on dynamic environments. In Proceedings of the GECCO, Washington, DC, USA (pp. 289-296).

Decanini, J. G. M. S., Tonelli-Neto, M. S., Malange, F. C. V., \& Minussi, C. R. (2011). Detection and classification of voltage disturbances using a fuzzy-ARTMAP-wavelet network. Electric Power Systems Research, 81(12), 2057-2065.

Dongli, X., Meng, X., \& Song, S. (2011). Study on technology system of self-healing control in smart distribution grid. In International conference on advanced power system automation and protection (pp. 1-5).

Dubé, L. (1996). How to use MODELS-based user-defined network components in ATP. In Proceedings European EMTP users group meeting, Budapest, Siegen (pp. 10-12). EEUG.

EMTP-RV. (2011). Eletromagnetic transient program. France: Powersys-Solutions, Le Puy-Sainte-Réparade.

Forrest, S., Perelson, A., Allen, L., \& Cherukuri, R. (1994). Self-nonself discrimination in a computer. In Proceedings of IEEE symposium on research in security and privacy (pp. 202-212).

Gungor, V. C., Sahin, D., Kocak, T., Ergut, S., Buccella, C., Cecati, C., \& Hancke, G. P. (2011). Smart grid technologies: communication technologies and standards. IEEE Transactions on Industrial Informatics, 7(4), 529-540.

LaPSEE. (2011). Practical 134 bus feeder data lines. Transactions of the AIEE, Part III - Power Apparatus and Systems, 76, 518-539 Available at: http://www.dee.feis. unesp.br/lapsee/.

Lima, F. P. A., Lotufo, A. D. P., \& Minussi, C. R. (2013). Artificial immune systems applied to voltage disturbance diagnosis in distribution electrical systems. In PowerTech-2013, Grenoble, France (pp. 1-6).

Lima, F. P. A., Lotufo, A. D. P., \& Minussi, C. R. (2014). Disturbance detection for optimal database storage in electrical distribution systems using artificial immune systems with negative selection. Electric Power Systems Research, 109, 54-62. 
Lima, F. P. A., Lotufo, A. D. P., \& Minussi, C. R. (2015). Wavelet-artificial immune system algorithm applied to voltage disturbance diagnosis in electrical distribution systems. IET Generation, Transmission \& Distribution, 9, 1104-1111.

Lima, F. P. A., Minussi, C. R., Bessa, R. B., \& Fidalgo, J. N. (2015). A modified negative selection algorithm applied in the diagnosis of voltage disturbances in distribution electrical systems. In Proceedings of the 18th international conference on intelligent system application to power systems (ISAP), Porto, Portugal (pp. 1-6).

Lima, F. P. A., \& Minussi, C. R. (January-2014). Voltage disturbance analysis of electrical power systems based on artificial immune system with continuous-learning (pp. 1-12). Brazil: Internal Publication, Electrical Engineering Department, UNESP, Univ Estadual Paulista, Ilha Solteira (SP) (in Portuguese).

Marchiori, S. C., Silveira, M. C. G., Lotufo, A. D. P., Minussi, C. R., \& Lopes, M. L. M. (2011). Neural network based on adaptive resonance theory with continuous training for multi-configuration transient stability analysis of electric power systems. Applied Soft Computing, 11, 706-715.

MATLAB (2011). 7.8 Version, Mathworks Company.

Northcote-Green, J., \& Wilson, R. (2007). Control and automation of electrical power distribution systems. Boca Raton: CRC/Taylor \& Francis.
Oleskovicz, M., Coury, D. V., Felho, O. D., Usida, W. F., Carneiro, A. F. M., \& Pires, L. R. S. (2009). Power quality analysis applying a hybrid methodology with wavelet transform and neural networks. International Journal of Electrical Power E' Energy Systems, 31(5), 206-212.

Pham, M.-T., \& Cham, T.-J. (2007). Online learning asymmetric boosted classifiers for object detection. In Proceedings of the CVPR (pp. 1-6).

Uyar, M., Yildririm, S., \& Gencoglu, M. T. (2008). An effective wavelet-based feature extraction method classification of power quality disturbance signals. Electric Power System Research, 78(10), 1747-1755.

Wylie, C. S., \& Shakhnovich, E. I. (2012). Mutation induced extinction in finite populations: lethal mutagenesis and lethal isolation. PLoS Computational Biology, 8, $1-6$.

Wylie, M., \& Pleydell, M. (2008). Network management data acquisition and analysis. In Road transport information and control - RTIC 2008, United Kingdom (pp. 1-3)

Zhang, M., Li, K., \& Hu, Y. (2011). A real-time classification method of power quality disturbances. Electric Power System Research, 81(12), 660-666. 